\title{
ÁGUA DE COCO EM PÓ COMO SUPLEMENTO HIDROELETROLÍTICO E ENERGÉTICO PARA ATLETAS
}

\author{
POWDERED COCONUT WATER AS HYDROELECTROLYTIC AND ENERGETIC \\ SUPPLEMENT FOR ATHLETES \\ AGUA DE COCO EN POLVO COMO SUPLEMENTO HIDROELECTROLITICO \\ Y ENERGÉTICO PARA ATLETAS
}

Artigo Original OrIGINAL ARTICLE Artículo Original

\begin{abstract}
Antônio Carlos Lopes Pinto' (Profissional de Educação Física)

Mauro Regis Vieira ${ }^{3}$

(Agrônomo)

Danilo Lopes Ferreira Lima² (Profissional de Educação Física)

Francisco de Assis Francelino Alves ${ }^{3}$ (Profissional de Educação Física)

Ricardo Lima dos Santos ${ }^{2}$

(Profissional de Educação Física)

1. Universidade Estadual do Ceará. Fortaleza, Ceará, Brasil.

2. Universidade de Fortaleza,

Fortaleza, Ceará, Brasil.

3. Universidade Federal do Ceará.

Fortaleza, Ceará, Brasil.
\end{abstract}

\section{Correspondência:}

Av. Engenheiro Leal Lima Verde, 679. 60833-175. Fortaleza, CE, Brasil acarloslopes@yahoo.com.br

\section{RESUMO}

Introdução: O uso de bebidas esportivas vem sendo adotado em diversas modalidades com o intuito de propiciar hidratação adequada durante atividades físicas. A água do coco verde vem sendo usada como repositor de eletrólitos devido à sua composição físico-química rica em sais minerais. Objetivo: Avaliar uma bebida esportiva à base de água de $\mathrm{Coco}$ em pó $\left(\mathrm{ACP}{ }^{\circledR}\right)$ a partir de um blend de $\mathrm{ACP}{ }^{\circledR} \mathrm{Com}$ polpa de maracujá (Passiflora edulis S.) voltada ao mercado de alimentos funcionais, com o objetivo de melhorar o desempenho de atletas amadores e profissionais em relação a um produto repositor eletrolítico já estabelecido no mercado (Gatorade ${ }^{\circledR}$ sabor maracujá). Métodos: 0 experimento obedeceu a um delineamento inteiramente casualizado em esquema fatorial $2 \times 2 \times 3$ ( 2 gêneros $\times 2$ tipos de bebidas $\times 3$ dias da semana), comparando o desempenho de dois grupos de atletas, sendo cinco atletas do sexo masculino e cinco atletas do sexo feminino, em relação à eficácia de dois suplementos hidroeletrolíticos $\left(A C P^{\circledR} \operatorname{com} 20 \%\right.$ de polpa de maracujá e Gatorade ${ }^{\circledR}$ sabor maracujá) em diferentes dias da semana (segunda-feira, quarta-feira e sexta-feira). Resultados: ACP ${ }^{\circledR}$ com polpa de maracujá apresentou resultados semelhantes ao Gatorade ${ }^{\circledR}$ sabor maracujá em relação à osmolaridade plasmática do sangue dos atletas, os quais não apresentaram nenhuma perda de desempenho e tiveram maior conforto gástrico ao se hidratarem com $\mathrm{ACP}{ }^{\circledR} \mathrm{Com}$ polpa de maracujá. Conclusão: As respostas fisiológicas mostram a eficiência de $A C P{ }^{\circledR}$ Com polpa de maracujá como um repositor de eletrólitos semeIhante ao do produto Gatorade ${ }^{\circledR}$ sabor maracujá, podendo assim ACP ${ }^{\circledR}$ com polpa de maracujá também ser utilizada como uma bebida esportiva.

Palavras-chave: bebidas isotônicas, hidratação, desempenho atlético, atletismo, osmolaridade.

\section{ABSTRACT}

Introduction: The use of sports drinks has been adopted in various forms in order to provide adequate hydration during physical activities. Green coconut water has been used as electrolyte replenishing due to its physicochemical composition, which is rich in minerals. Objective: To evaluate a sports drink based on powdered coconut water (ACP $\left.{ }^{\circledR}\right)$ as from a blend of $A C P{ }^{\circledR}$ with passion fruit pulp (Passiflora edulis S.), focused on the functional food market, aiming at improving the performance of amateur and professional athletes compared with an electrolytic replenishing already established in the market (passion fruit flavored Gatorade ${ }^{\circledast}$ ). Methods: The experiment followed a completely randomized design in a factorial scheme $2 \times 2 \times 3$ (2 genders $\times 2$ drink types $\times 3$ times a week) comparing the performance of two groups of athletes, five male athletes and five female athletes, as to the effectiveness of two hydroelectrolytic supplements (ACP ${ }^{\circledR}$ with 20\% passion fruit pulp and passion fruit flavored Gatorade ${ }^{\circledast}$ ) on different days of the week (Monday, Wednesday and Friday). Results: ACP ${ }^{\circledR}$ with passion fruit pulp showed results similar to the passion fruit flavored Gatorade ${ }^{\circledR}$ in relation to blood plasma osmolarity of athletes, who experienced no loss of performance and more gastric comfort when hydrated with $A C P^{\circledR}$ with passion fruit pulp. Conclusion: The physiological responses show the efficiency of $A C P^{\circledR}$ with passion fruit pulp as a replenishing of electrolytes similar to Gatorade ${ }^{\circledR}$ passion fruit flavored, the $A C{ }^{\circledR}$ with passion fruit pulp being, therefore, also appropriate to be used as a sports drink.

Keywords: energy drinks, hydration, athletic performance, athletics, osmolality.

\section{RESUMEN}

Introducción: El uso de bebidas deportivas viene siendo adoptado en diversas modalidades con el objetivo de propiciar hidratación adecuada durante actividades físicas. El agua de coco verde viene siendo usada como repositor de electrolitos debido a su composición físico-química rica en sales minerales. Objetivo: Evaluar una bebida deportiva a base de agua de coco en polvo $\left(A C P^{\circledR}\right)$ a partir de un blend de $A C P^{\circledR}$ con pulpa de maracuyá (Passiflora edulis S.) dirigida al mercado de alimentos funcionales, con el objetivo de mejorar el desempeño de atletas amateurs y profesionales en relación a un producto repositor electrolítico ya establecido en el mercado (Gatorade ${ }^{\circledast}$ sabor maracuyá). Métodos: El experimento obedeció a una delineación totalmente casualizada en esquema factorial $2 \times 2 \times 3$ ( 2 géneros $\times 2$ tipos de bebidas $\times 3$ días de la semana), comparando el desempeño de dos grupos de atletas, siendo cinco atletas del sexo masculino y cinco atletas del sexo femenino, en relación a la eficacia de dos suplementos hidroelectrolíticos $\left(A C P^{\circ}\right.$ 
con 20\% de pulpa de maracuyá y Gatorade ${ }^{\circledR}$ sabor maracuyá) en diferentes días de la semana (lunes, miércoles y viernes). Resultados: ACP ${ }^{\circledR}$ con pulpa de maracuyá presentó resultados semejantes al Gatorade ${ }^{\circledR}$ sabor maracuyá en relación a la osmolaridad plasmática de la sangre de los atletas, los que no presentaron ninguna pérdida de desempeño y tuvieron mayor comodidad gástrica al hidratarse con $A C{ }^{\circledR}$ con pulpa de maracuyá. Conclusión: Las respuestas fisiológicas muestran la eficiencia de $A C P^{\circledR}$ con pulpa de maracuyá como un repositor de electrolitos semejante al del producto Gatorade ${ }^{\circledR}$ sabor maracuyá, pudiendo así ACP ${ }^{\circledR}$ con pulpa de maracuyá también ser utilizada como una bebida deportiva.

Palabras clave: bebidas isotónicas, hidratación, desempeño atlético, atletismo, osmolaridad.

\section{INTRODUÇÃO}

O processo de desidratação é um dos fatores que aumenta os níveis de estresse causado pelo exercício, pois eleva a temperatura do corpo, torna as respostas fisiológicas menos expressivas, prejudica o desempenho físico e deixa o organismo mais suscetível a doenças'.

O uso de bebidas isotônicas, também conhecidas como bebidas esportivas, vem sendo adotado por atletas de diversas modalidades com o intuito de propiciar uma hidratação adequada durante atividades físicas de alta intensidade.

A água do coco verde vem sendo usada como uma alternativa de repositor de eletrólitos durante sessões de atividades físicas devido à sua composição físico-química que é rica em sais minerais, aminoácidos, proteínas, vitaminas e minerais².

Em maiores níveis de desidratação há o aumento da temperatura esofágica que sinaliza para a redução da sudorese, levando a aumento na osmolaridade do plasma sanguíneo e na concentração sérica de sódio. Isto sugere que uma importante meta da ingestão de líquidos durante o exercício é prevenir variações na osmolaridade e na concentração plasmática de sódio ${ }^{3}$.

Segundo Brito ${ }^{4}$, a reposição tanto hídrica quanto de nutrientes é uma necessidade que está relacionada diretamente com a intensidade e duração do exercício e com a temperatura do local da prática.

A avaliação do estado de hidratação corporal antes, durante e após o exercício físico é de fundamental importância por ser um fator determinante de desempenho para a prática de atividades físicas principalmente para a prática constante, além de ajudar a prevenir ou evitar os problemas de saúde provocados pelo estado de desidratação ${ }^{5}$. 0 principal método de avaliação do estado de hidratação corporal em nível de práticas laboratoriais é pela osmolaridade plasmática, onde exatidão na medida é exigida ${ }^{3}$. Assim, para avaliação do estado de hidratação dos indivíduos ativos tem sido considerada como um bom método não invasivo a gravidade específica da urina ${ }^{6}$. Um método prático de avaliação do estado de hidratação é pela variação do peso corporal, onde é verificado o peso corporal antes e após o exercício e a partir da diferença destes é possível calcular o percentual de perda de peso para classificar o estado de hidratação ${ }^{7}$.

Quanto ao conteúdo do plasma sanguíneo, no homem saudável, a osmolaridade plasmática é mantida dentro de limites fisiológicos muito estreitos, entre 280-295mOsm/kg, pela integração, com sucesso, da ingestão de água e excreção, sendo a última controlada principalmente pelas ações antidiuréticas da hormona de origem neurohipofisária, vasopressina ${ }^{8}$. Este processo é conhecido como osmorregulação e é tão eficaz que em condições fisiológicas, não permite que a osmolaridade plasmática varie mais do que 1 a $2 \%$.

Quando o homem é sujeito à privação de água, há um aumento na osmolaridade plasmática, o qual é detectado por células especializadas osmossensíveis localizadas no hipotálamo anterior ${ }^{10}$.

Em relação aos indicadores sanguíneos são apontadas a concentra- ção de hemoglobina, o hematócrito (que sofre alterações com a postura o que implica a estandardização da mesma antes da colheita de sangue) e a concentração de sódio e a osmolaridade do sangue (a qual aumenta na hipohidratação), mas já têm sido verificadas perdas de mais de 3\% da massa corporal, pela sudação intensa, sem se verificar alteração de hematócrito e na osmolaridade ${ }^{11}$.

A reposição de eletrólitos é de fundamental importância para o balanço osmótico das células. Em atletas, a reposição de eletrólitos precisa ser feita antes, durante e depois dos treinos. Essa reposição deve ser feita de forma rápida e eficiente, por produtos específicos, tais como as bebidas esportivas, para que haja a manutenção do balanço osmótico das células e os atletas não percam rendimento durante a atividade física ${ }^{12}$.

A água de coco é utilizada na cultura popular como substituto da água, e também para repor eletrólitos nos casos de desidratação ${ }^{13}$. 0 sódio, o principal cátion do fluido extracelular, regula o tamanho do compartimento celular, bem como o volume do plasma sanguíneo e auxilia na condução de impulsos nervosos e no controle da contração muscular. O potássio, o principal cátion do fluido intracelular, promove o crescimento celular, estando presente em pequenas quantidades no fluido extracelular e, juntamente com o sódio, participa da manutenção do equilíbrio hídrico normal. Já o cálcio é importante na regulação da atividade neuromuscular ${ }^{14}$.

Saat et al. ${ }^{15}$ afirmam que a ingestão de água de coco pode ser utilizada para reidratação após exercícios físicos. A água de coco pode representar um produto concorrente às bebidas para o esporte, devido a sua capacidade de repor eletrólitos ${ }^{16}$.

Eletrólitos em soluções isotônicas são mais rapidamente absorvidos restabelecendo prontamente as perdas de nutrientes. Nutrientes como o sódio e o potássio devem estar presentes na composição das bebidas isotônicas, a fim de possibilitar a recuperação das perdas de sódio e potássio através da urina e da pele. A água de coco apresenta em sua composição estes componentes ${ }^{13}$ e, mesmo não sendo rica em vitaminas, contém ácido ascórbico (vitamina C) e vitaminas do complexo $\mathrm{B}^{17}$.

A composição básica da água de coco é de $93 \%$ de água, $4 \%$ de carboidratos, 0,1\% de gorduras, 0,02\% de cálcio, 0,01\% de fósforo, 0,5\% de ferro, além de aminoácidos, vitamina $C$ e vitaminas do complexo $B^{18}$. Os cátions predominantes são potássio, cálcio e magnésio. Sódio, cloro e fosfato são encontrados em menores concentrações ${ }^{19}$. Esta solução hipotônica é mais ácida do que o plasma sanguíneo ${ }^{20}$.

A água de coco vem sendo utilizada por atletas de alto desempenho como um isotônico natural devido sua alta osmolaridade, altos níveis de carboidratos, principalmente, frutose e glicose e é rica em aminoácidos, tais como lisina, cistina, fenilalanina, histidina e triptofano².

Os isotônicos, ou bebidas esportivas, são bastante consumidos pela população, principalmente por atletas profissionais e por pessoas que praticam esportes, com uma frequência que varia de 12\% a 27,7\%21.

As bebidas esportivas possuem essa denominação por apresentar osmolaridade similar à do sangue humano. Todavia, a despeito de suas 
propriedades hidroeletrolíticas, as mesmas podem apresentar efeitos danosos sobre os dentes, os rins e a mucosa gástrica devido ao seu baixo pH e presença de ácido cítrico em sua composição ${ }^{22}$. Essas características fazem com que apresentem elevado potencial erosivo aos tecidos duros dentais, especialmente quando consumidas de forma excessiva e/ou por períodos prolongados ${ }^{23}$. Entretanto, a água de coco verde, sendo um produto natural, tem suas benesses estabelecidas e comprovadas para todo o sistema digestório humano, sendo um excelente repositor de sais minerais ${ }^{1}$.

A água de coco em pó tem como insumo básico o líquido endospérmico do coco que, em sua forma processada, confere estabilidade e longevidade de prateleira, sem problemas de acondicionamento e supera a toda e qualquer outra tecnologia de conservação, uma vez que mantém as propriedades inerentes do produto original. Daí a grande vantagem da água de coco em pó. Uma vez processada, não modifica sua composição até sua utilização, garantindo um padrão confiável.

O objetivo desse estudo foi avaliar uma bebida esportiva à base de água de coco em pó $\left(A C P^{\circledR}\right)$ a partir de um blend de $A C{ }^{\circledR}$ com polpa de maracujá (Passiflora edulis S.) voltada ao mercado de alimentos funcionais com o objetivo de melhorar o desempenho de atletas amadores e profissionais em relação a um produto repositor eletrolítico já estabelecido no mercado (Gatorade ${ }^{\circledR}$ sabor maracujá).

\section{MÉTODOS}

O produto $A C{ }^{\circledR}$ com frutas tropicais foi produzido pela empresa $A C P^{\circledR}$ Biotecnologia (Fortaleza, Ceará). A matéria-prima utilizada foi padronizada quanto ao estado de maturação dos frutos e certificada como produto orgânico pelo Instituto Biodinâmico (IBD). Os frutos foram processados seguindo as normas de Boas Práticas de Fabricação da Agência Nacional de Vigilância Sanitária (ANVISA).

O estudo iniciado após a aprovação pelo Comitê de Ética em Pesquisa da Universidade de Fortaleza, Fortaleza, Ceará, Brasil (UNIFOR) sob o n 301/2007.

O experimento obedeceu a um delineamento Inteiramente casualizado em esquema fatorial 2 vs. 2 vs. 3 (2 gêneros $x 2$ tipos de bebidas vs. 3 dias da semana) com cinco repetições.

Foi comparado o desempenho de dois grupos de atletas, sendo cinco atletas do sexo masculino e cinco atletas do sexo feminino, em relação à eficácia de dois suplementos hidroeletrolíticos $\left(A C P{ }^{\circledR} \mathrm{Com}\right.$ $20 \%$ de polpa de maracujá e Gatorade ${ }^{\circledR}$ sabor maracujá) em diferentes dias da semana (segunda-feira, quarta-feira e sexta-feira).

Cada atleta constituiu uma unidade experimental, sendo cinco atletas do sexo masculino e cinco atletas do sexo feminino, com média de

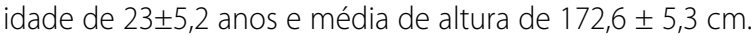

A administração dos suplementos hidroeletrolíticos seguiu as recomendações do American College of Sports Medicine ${ }^{4}$, sendo os mesmos mantidos a temperatura entre 15 e $20^{\circ} \mathrm{C}$.

O protocolo preliminar de hidratação foi realizado em duas etapas, cada qual com fornecimento de $250 \mathrm{~mL}$ de suplemento hidroeletrolítico. A primeira etapa consistiu no fornecimento dos suplementos duas horas antes dos testes e a segunda etapa no fornecimento dos suplementos 30 minutos antes dos testes, perfazendo $500 \mathrm{~mL}$ de suplemento hidroeletrolítico administrado antes do treinamento. Durante a realização dos testes os atletas ingeriram $100 \mathrm{~mL}$ de suplemento a cada 10 minutos de exercício, perfazendo $600 \mathrm{~mL}$. Os treinamentos foram realizados durante os dias em que os dados foram coletados e tiveram alta intensidade, havendo a necessidade dos mesmos ocorrerem em condições semelhantes de velocidade do vento, temperatura e umidade relativa do ar, no horário de 09 horas às 10 horas da manhã. As medidas antropométricas de peso e altura foram realizadas com a utilização de uma balança digital da marca
Plenna (Plebal Importação/Exportação Ltda. - USA) e um estadiômetro profissional marca Sanny - referência ES2020 (América Medical do Brasil), devidamente calibrados. As pesagens foram realizadas antes e após os treinos, com o avaliado em posição ortostática, de costas para a balança e com os pés afastados lateralmente. No procedimento da medida da altura, o avaliado ficou também na posição ortostática em apneia inspiratória, ereto, braços estendidos ao longo do corpo e pés unidos.

As amostras de sangue foram colhidas, após os treinos, através de um profissional da área de saúde, ou seja, um enfermeiro, inscrito no Conselho Regional de Enfermagem do Ceará (COREN). Através do sangue coletado foram verificadas as diferenças no volume plasmático e na osmolalidade sérica. A partir deste material foi só analisada a osmolaridade plasmática na corrente sanguínea dos atletas. 0 procedimento obedeceu todas as normas de biossegurança, todos os participantes assinaram o Termo de Consentimento Livre e Esclarecido, e foram observados os preceitos de ética estabelecidos pela resolução de no 196/96 do Conselho Nacional de Saúde, sobre pesquisa envolvendo seres humanos, através do uso de materiais descartáveis e de equipamentos de proteção individual. Os exames laboratoriais foram realizados pelo Laboratório de Análises Clínicas do Núcleo de Atenção Médica Integrada (NAMI) da Universidade de Fortaleza (UNIFOR).

Os dados experimentais foram submetidos à análise de variância ao nível de $1 \%$ e 5\% de probabilidade e Teste de Tukey a 5\% de probabilidade. Como ferramenta de auxílio às análises estatísticas, adotou-se o procedimento do programa SAEG/UFV24.

\section{RESULTADOS E DISCUSSÃO}

Os valores de osmolaridade plasmática na corrente sanguínea dos atletas variaram significativamente ao nível de $1 \%$ em relação ao fator gênero (sexo masculino e sexo feminino) e ao nível de 5\% em relação ao fator dia da semana (segunda-feira, quarta-feira e sexta-feira), conforme apresentado na tabela 1.

Os atletas do sexo masculino apresentaram nível médio de osmolaridade plasmática significativamente maior quando comparados com os atletas do sexo masculino (figura 1).

O aumento significativo na osmolaridade plasmática nas amostras de sangue dos atletas do sexo masculino indica uma provável pré-desidratação em comparação aos valores obtidos em atletas do sexo feminino. Este resultado pode ser inerente à maior taxa de transpiração de atletas do sexo masculino devido ao estresse ambiental durante o período de atividade física ${ }^{3}$.

Atletas do sexo masculino apresentam taxa de sudorese superior aos atletas do sexo feminino e, consequentemente, apresentam aumentos na osmolaridade do plasma sanguíneo, necessitando uma maior ingestão

Tabela 1. Quadro de Análise de Variância com a significância estatística do Teste de Fisher para a osmolaridade plasmática $\left(\mathrm{mOsm} / \mathrm{kg} \mathrm{H}_{2} \mathrm{O}\right)$ presente em amostras de sangue de atletas submetidos a ingestão de água de coco em pó (ACP $\left.{ }^{\circledR}\right)$ com polpa de maracujá e Gatorade ${ }^{\circledR}$ sabor maracujá antes e após treinamento intensivo e em diferentes dias da semana, Fortaleza, Ceará, 2007.

\begin{tabular}{c|c|c|c}
\hline Fonte de Variação & GL & $\begin{array}{c}\text { Quadrado } \\
\text { médio }\end{array}$ & $\mathbf{F}$ \\
\hline Gênero & 1 & 186,9135 & $112,25^{* *}$ \\
\hline Tipo de bebida & 1 & 0,4335 & $0,26^{\text {ns }}$ \\
\hline Dia da semana & 2 & 8,8145 & $4,93^{*}$ \\
\hline Interação Gênero x Tipo de bebida & 1 & 0,9375 & $0,56^{\text {ns }}$ \\
\hline Interação Gênero x Dia da semana & 2 & 4,7285 & $2,84^{\text {ns }}$ \\
\hline Interação Tipo de bebida x Dia da semana & 2 & 1,0355 & $0,63^{\text {ns }}$ \\
\hline Resíduo Gênero x Tipo de bebida x Dia da semana & 2 & 3,7815 & $2,27^{\text {ns }}$ \\
\hline CV (\%) & 48 & 1,6651 & \\
\hline
\end{tabular}

*Significativo pelo teste $\mathrm{F}$ a 5\%; * ${ }^{*}$ Significativo pelo teste $\mathrm{F}$ a $1 \%$; ns: não significativo. 


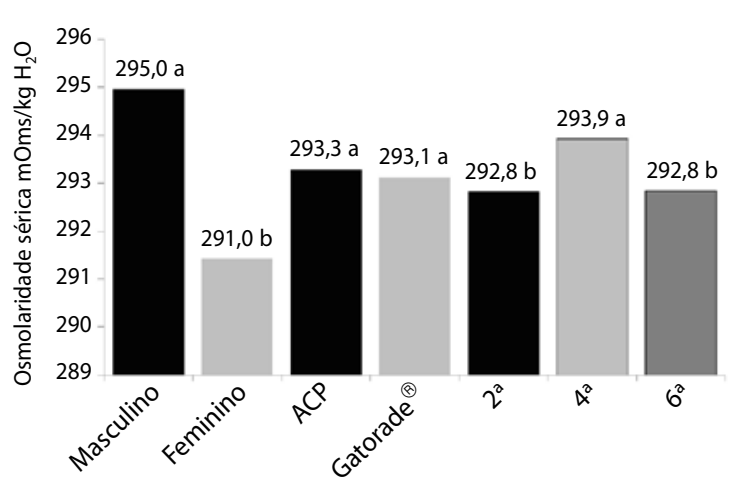

Médias com letras iguais não diferem significativamente pelo Teste de Tukey ao nível de $5 \%$ de probabilidade. Diferença mínima significativa (dms): Gênero: 0,670;Tipo de bebida: 0,670; e, dia da semana: 0,987.

Figura 1. Valores médios dos níveis de osmolaridade plasmática em atletas dos sexos masculino e feminino submetidos à ingestão de água de coco em pó $\left(A C P^{\circledR}\right)$ com polpa de maracujá e Gatorade ${ }^{\circledR}$ sabor maracujá após treino intensivo, em diferentes dias de treinamento, Fortaleza, Ceará, 2007.

de líquidos durante os treinamentos para manter os níveis normais ${ }^{3}$, contribuindo para a otimização do desempenho nos treinos ${ }^{4}$.

Efeitos de pré-desidratação também foram relatados em experimento onde houve a imposição de exercícios físicos em atletas sob condições de estresse ambiental, resultando na homeostasia do equilíbrio hídrico, porém com comprometimento no desempenho dos atletas $^{25}$. Essas respostas fisiológicas podem ser apoiadas na significância do Teste de Fisher para o fator "Dia da semana", o qual apresentou diferença significativa ao nível de 5\% (tabela 1).

Os fatores externos (temperatura, umidade relativa do ar e velocidade do vento), durante o período de treinos no dia de quarta-feira, influenciaram negativamente no desempenho dos atletas de ambos os sexos, independente da bebida esportiva consumida, aumentando os níveis de osmolaridade plasmática no sangue e promovendo uma diferença significativa ao nível de $5 \%$ em relação aos outros dias de treinamento (figura 1). Provavelmente esses resultados influenciaram na termorregulação dos atletas do sexo masculino e em suas taxas de transpiração. Alterações ocorridas em atletas de alto nível podem ser causadas por fatores externos, reduzindo o desempenho e comprometendo a saúde dos atletas no decorrer dos treinos ${ }^{25}$.

Vale ressaltar que os valores de osmolaridade plasmática dos atletas, tanto do sexo masculino quanto do sexo feminino, durante todos os dias de treinamento intensivo ficaram dentro dos limites fisiológicos considerados normais, independente da ingestão de $\mathrm{ACP}{ }^{\circledR}$ ou de Gatorade ${ }^{\circledast}$ (figura 1), sendo que os valores considerados normais estão entre 280 e $295 \mathrm{mOsm} / \mathrm{kg}^{8}$.

As repostas fisiológicas baseadas nos níveis de osmolaridade plasmática obtidas nas amostras de sangue colhidas dos atletas mostram que a eficiência da $\mathrm{ACP}^{\circledR} \mathrm{com}$ polpa de maracujá como um repositor de eletrólitos para atletas de alto nível não diferiu estatisticamente com relação à eficiência do produto Gatorade ${ }^{\circledast}$ sabor maracujá, podendo assim, a $\mathrm{ACP}^{\circledR}$ com polpa de maracujá também ser utilizada como uma bebida esportiva.

\section{Variação da massa corporal $\left(\Delta_{M}\right)$}

Os valores de massa corporal em atletas dos sexos masculino e feminino submetidos à ingestão de água de coco em pó $\left(\mathrm{ACP}{ }^{\circledR}\right) \mathrm{Com}$ polpa de maracujá e Gatorade ${ }^{\circledR}$ sabor maracujá foram mensurados antes e após os treinos, realizados em diferentes dias, conforme tabela 2. A diferença de massa corporal passou por transformação dos valores mensurados para realização da Análise de Variância aos níveis de 5\% e de 1\% e Teste de Tukey ao nível de $5 \%{ }^{24}$.
Os valores referentes à diferença média de massa corporal $\left(\Delta_{M}\right)$ foram significativos, ao nível de $1 \%$ pelo Teste de Fisher, com relação ao tipo de bebida ingerida pelos atletas durante o período de coleta dos resultados (tabela 3).

A avaliação do estado de hidratação corporal dos atletas submetidos a intensivos treinamentos foi feita a partir da diferença de massa corporal, sendo que os atletas que ingeriram a $\mathrm{ACP}^{\circledR} \mathrm{com}$ polpa de maracujá, independente do gênero ou do dia da semana, apresentaram uma maior diferença de massa corporal quando comparados com os atletas que consumiram Gatorade ${ }^{\circledR}$ sabor maracujá (figura 2).

Tabela 2. Valores médios de massa corporal e diferença média de massa corporal $(\triangle M)$ em atletas dos sexos masculino e feminino submetidos à ingestão de água de coco em pó $\left(A C P{ }^{\circledR}\right)$ com polpa de maracujá e Gatorade ${ }^{\circledR}$ sabor maracujá mensurados antes e após os treinos, em diferentes dias, Fortaleza, Ceará, 2007.

\begin{tabular}{c|c|c|c|c|c|c}
\hline \multirow{4}{*}{ Gênero } & \multicolumn{3}{|c|}{ ACP $^{\circledR}$} & \multicolumn{3}{c}{ Gatorade $^{\circledR}$} \\
\cline { 2 - 7 } & $\begin{array}{c}\text { Massa } \\
\text { antes (kg) }\end{array}$ & $\begin{array}{c}\text { Massa } \\
\text { depois (kg) }\end{array}$ & $\mathbf{\Delta M}$ & $\begin{array}{c}\text { Massa } \\
\text { antes (kg) }\end{array}$ & $\begin{array}{c}\text { Massa } \\
\text { depois (kg) }\end{array}$ & \multicolumn{1}{c}{$\boldsymbol{\Delta}$} \\
\hline \multirow{5}{*}{ Masculino } & 68,5 & 68,5 & 0,0 & 67,9 & 68,3 & 0,4 \\
\cline { 2 - 7 } & 61,0 & 60,8 & $-0,2$ & 60,5 & 60,3 & $-0,2$ \\
\cline { 2 - 7 } & 57,5 & 57,7 & 0,2 & 57,0 & 57,4 & 0,4 \\
\cline { 2 - 7 } & 65,7 & 65,2 & $-0,5$ & 64,2 & 63,9 & $-0,3$ \\
\cline { 2 - 7 } & 60,6 & 60 & $-0,6$ & 60,5 & 60,2 & $-0,3$ \\
\hline \multirow{5}{*}{ Feminino } & 66,1 & 65,9 & $-0,2$ & 66,8 & 66,2 & $-0,6$ \\
\cline { 2 - 7 } & 61,2 & 61,8 & 0,6 & 60,9 & 60,9 & 0,0 \\
\cline { 2 - 7 } & 65,1 & 64,7 & $-0,4$ & 64,1 & 63,9 & $-0,2$ \\
\cline { 2 - 7 } & 57,0 & 56,9 & $-0,1$ & 57,2 & 57,0 & $-0,2$ \\
\cline { 2 - 7 } & 53,0 & 52,6 & $-0,4$ & 53,3 & 53,0 & $-0,3$ \\
\hline
\end{tabular}

Tabela 3. Quadro de Análise de Variância com a significância estatística do Teste de Fisher para a diferença de massa corporal $(\Delta M)$ em atletas submetidos à ingestão de água de coco em pó $\left(A C P^{\circledR}\right)$ com polpa de maracujá e Gatorade ${ }^{\circledR}$ sabor maracujá antes e após treinamento intensivo em diferentes dias, Fortaleza, Ceará, 2007.

\begin{tabular}{|c|c|c|c|}
\hline Fonte de Variação & GL & $\begin{array}{c}\text { Quadrado } \\
\text { Médio }\end{array}$ & $\mathbf{F}$ \\
\hline Gênero & 1 & 186,9135 & $2,55^{\mathrm{ns}}$ \\
\hline Tipo de bebida & 1 & 0,4335 & $13,57^{* *}$ \\
\hline Dia da semana & 2 & 8,8145 & $2,98^{\mathrm{ns}}$ \\
\hline Interação Gênero x Tipo de bebida & 1 & 0,9375 & $0,77^{\mathrm{ns}}$ \\
\hline Interação Gênero x Dia da semana & 2 & 4,7285 & $3,97^{*}$ \\
\hline Interação Tipo de bebida $\times$ Dia da semana & 2 & 1,0355 & $0,65^{\mathrm{ns}}$ \\
\hline Interação Gênero x Tipo de bebida x Dia da semana & 2 & 3,7815 & $0,55^{\mathrm{ns}}$ \\
\hline Resíduo & 48 & 1,6651 & \\
\hline \multicolumn{3}{|l|}{ CV (\%) } & 22,00 \\
\hline
\end{tabular}

*Significativo pelo teste $\mathrm{F}$ a $5 \%$; ** Significativo pelo teste $\mathrm{F}$ a 1\%; ns: não significativo.

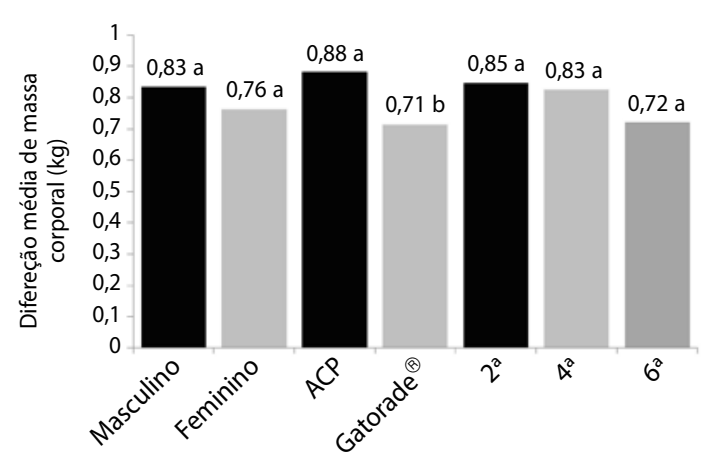

Fonte: Autores, 2007. Médias com letras iguais não diferem significativamente pelo Teste de Tukey ao nível de $5 \%$ de probabilidade. Diferença mínima significativa (dms): Gênero: 0,912; Tipo de bebida: 0,912 ; e, dia da semana: 0,134 .

Figura 2. Valores médios de diferença de massa corporal em atletas dos sexos masculino e feminino submetidos à ingestão de água de coco em pó (ACP $\left.{ }^{\circledR}\right)$ com polpa de maracujá e Gatorade ${ }^{\circledR}$ sabor maracujá antes e durante treinamento intensivo, em diferentes dias de treinamento, Fortaleza, Ceará, 2007. 
Esses resultados provavelmente se caracterizam pela maior perda de líquidos (suor) por parte de atletas que consumiram $A C{ }^{\circledR}$ com polpa de maracujá devido uma insuficiente reposição de eletrólitos durante os treinos, como em ensaios conduzidos por Shirreffs ${ }^{11}$, onde foi verificada perda de mais de 3\% da massa corporal, pela sudação intensa, sem se verificar alteração na osmolaridade plasmática de amostras de sangue dos atletas.

A diferença de massa corporal $\left(\Delta_{M}\right)$ foi influenciada significativamente ao nível de $5 \%$ pela interação dos fatores gênero (masculino e feminino) e dias da semana (segunda-feira, quarta-feira e sexta-feira) durante o experimento (tabela 4).

Os atletas do sexo feminino apresentaram menor diferença média de massa corporal em relação aos atletas do sexo masculino em ensaios conduzidos na quarta-feira (tabela 4). Esse comportamento se apresentou semelhante ao comportamento obtido nos níveis de osmolaridade plasmática nos atletas do sexo feminino em ensaio experimental, ou seja, menores valores de osmolaridade plasmática nas amostras de sangue colhidas em atletas do sexo feminino.

Maiores diferenças de perda de massa corporal em atletas do sexo masculino (tabela 4) reforçam a hipótese de pré-desidratação baseada em maiores concentrações de osmolaridade plasmática nos atletas do sexo masculino durante os treinos em função da manutenção do balanço hídrico (figura 2). Entretanto, Montain, Latzka e Sawka7 afirmaram que a regulação osmótica das células epidérmicas influencia na menor variação do peso corporal em função do estado de hidratação e do desempenho sem que haja variação na osmolaridade plasmática dos atletas para que haja manutenção e equilíbrio durante a atividade física e, consequentemente, menor perda de rendimento.

Mesmo com uma diferença significativa ao nível de 1\% com re-
Tabela 4. Valores médios das diferenças de massa corporal $(\Delta M)$ entre atletas dos sexos masculino e feminino submetidos à ingestão de água de coco em pó $\left(A C P{ }^{\circledR}\right)$ com polpa de maracujá e Gatorade ${ }^{\circledR}$ sabor maracujá mensurados antes e após os treinos, realizados em diferentes dias, Fortaleza, Ceará, 2007.

\begin{tabular}{c|c|c|c}
\hline \multirow{2}{*}{ Gênero } & \multicolumn{3}{|c}{ Dia da semana } \\
\cline { 2 - 4 } & Segunda-feira & Quarta-feira & Sexta-feira \\
\hline Masculino & $0,874 \mathrm{aA}$ & $0,879 \mathrm{aA}$ & $0,840 \mathrm{aA}$ \\
\hline Feminino & $0,822 \mathrm{aA}$ & $0,602 \mathrm{bB}$ & $0,862 \mathrm{aA}$ \\
\hline
\end{tabular}

a Nas linhas, médias transformadas com letra minúscula iguais não diferem ao nivel de $5 \%$. $\mathrm{B}$ Nas colunas médias transformadas com letras maiúsculas iguais não diferem ao nível de $5 \%$. Diferença mínima significativa (dms): Colunas: 0,158 e Linhas: 0,190.

lação aos valores médios na diferença de massa corporal em atletas que consumiram $A C{ }^{\circledR}$ com polpa de maracujá não foi constatada nenhuma influência no desempenho, independente do tipo de bebida consumida. No entanto, foi relato um maior conforto gástrico por parte dos atletas que se hidrataram com $A C{ }^{\circledR}$ com polpa de maracujá.

\section{CONCLUSÕES}

A água de coco em pó $\left(A C P^{\circledR}\right)$ com polpa de maracujá apresentou resultados semelhante ao Gatorade ${ }^{\circledR}$ sabor maracujá em relação à osmolaridade plasmática do sangue dos atletas.

O consumo de $A C P^{\circledR}$ com polpa de maracujá promoveu uma maior diferença média de massa corporal nos atletas do sexo feminino quando comparado ao Gatorade ${ }^{\circledR}$ sabor maracujá.

$\mathrm{O} A C{ }^{\circledR}$ com polpa de maracujá apresentou melhor conforto gástrico durante os treinamentos intensivos. As repostas fisiológicas mostram que a $A C{ }^{\circledR}$ com polpa de maracujá pode ser utilizada como bebida esportiva.

Todos os autores declararam não haver qualquer potencial conflito de interesses referente a este artigo.

\section{REFERÊNCIAS}

1. Meir R, Brooks $L$, Shield T. Body weight and tympanic temperature change in professional rugby league players during night and day games: a study in the field. J Strength Cond Res. 2003;17(3):566-72.

2. Gopikrishna V,Thomas T, Kandaswamy D. A quantitative analysis of coconut water: a new storage media for avulsed teeth. Oral Surg Oral Med Oral Pathol Oral Radiol Endod. 2008;105(2):e61-5.

3. American College of Sports Medicine, Sawka MN, Burke LM, Eichner ER, Maughan RJ, Montain SJ, et al. American College of Sports Medicine position stand. Exercise and fluid replacement. Med Sci Sports Exerc. 2007:39(2):377-90.

4. Brito IP. Considerações atuais sobre reposição hidroeletrolítica no esporte. Nutr Pauta 2003;11:48-52.

5. Carvalho T, Rodrigues T, Meyer F, Lancha JR. AH, De Rose EH. Modificações dietéticas, reposição hídrica, suplementos alimentares e drogas: comprovação de ação ergogênica e potenciais riscos para a saúde. Rev Bras Med Esporte. 2009;15(3):2-12.

6. Tirapegui J. Nutrição, metabolismo e suplementação na atividade física. São Paulo: Atheneu; 2005.

7. Montain SJ, Latzka, WA, Sawka MN. Control of thermoregulatory sweating is altered by hydration level and exercise intensity. J Appl Physiol. 1995;79(5):1434-9.

8. Greenleaf JE. Problem: thirst, drinking behavior, and involuntary dehydration. Med Sci Sports Exerc. 1992;24(6):645-56.

9. Johnson DR, Douglas D, Hauswald M, Tandberg D. Dehydration and orthostatic vital signs in women with hyperemesis gravidarum. Acad Emerg Med. 1995;2(8):692-7.

10. Hoffman JR, Maresh CM, Armstrong LE, Gabaree CL, Bergeron MF, Kenefick RW, et al. Effects of hydration state on plasma testosterone, cortisol and catecholamine concentrations before and during mild exercise at elevated temperature. Eur J Appl. Physiol Occup. Physiol. 1994;69(4):294-300.

11. Shirreffs SM. Markers of hydration status. J Sports Med Phys Fitness 2000;40(1):80-4.

12. Coyle EF, Hamilton MA. Fluid replacement during exercise: effects of physiological homeostasis and performance. In: Gisolfi CV, Lamb DR, editors. Fluid homeostasis during exercise: perspectives in exercise science and sports medicine. Indianapolis: Benchmark Press; 1990. p. 281-303.
13. Aragão WM, Isberner IV, Cruz EM. O. Água-de-coco. Aracaju: Embrapa; 2001.

14. Whitmire SJ. Água, eletrólitos e equilíbrio ácido-base. In: Mahan LK, Scott-Stump S. Krause: alimentos, nutrição e dietoterapia. São Paulo: Roca; 2002. p. 146-56.

15. Saat M, Singh R, Sirisinghe RG, Nawawi M. Rehydration after exercise with fresh young coconut water, carbohydrate-electrolyte beverage and plain water. J Physiol Anthropol Appl Human Sci. 2002;21 (2):93-104.

16. Agricultura 21. Enfoques: nueva bebida para el deporte: agua de coco. Rev FAO [accesso em 22 set 2013]1998. Disponible: http://www.fao.org/esp/revista/9810/sport3.htm

17. Atukorale DP. Goodness of tender coconut water. [acesso em 2013 Oct 11] 2001. Availabre from: URL http://www.island.1k/2001/12/26/feartur03

18. Aragão WMA. Importância do coqueiro-anão verde. [acesso em 11 out. 2013]. Disponível em: http:// www21.sede.embrapa.br/noticias/artigos/2000/artigo.2004-1207.2461636373/mostraartigo

19. Khan MN, Muti-Ur-Rehman, Khan KW. A study of chemical composition of Cocos nucifera L. (coconut) water and its usefulness as rehydration fluid. Pakistan J Bot. 2003;35:925-30.

20. Campbell-Falck D, Thomas T, Falck TM, Tutuo N, Clem K. The intravenous use of coconut water. Am J Emerg Med. 2000;18(1):108-11.

21. Zandim DL, Gilio C, Rossa Jr C, Sampaio JEC. Influência de bebidas isotônicas na remoção de smear layer de superfícies radiculares após raspagem. Estudo in vitro. Revista de Odontologia da UNESP. 2008;37(3):267-73.

22. Järvinen VK, Rytömaa II, Heinonen OP. Risk factors in dental erosion. J Dent Res. 1991;70(6):742-7.

23. Lussi A, Jaeggi T, Schärer S. The influence of different factors on in vitro enamel erosion. Caries Res. 1993;27(5):387-93.

24. Ribeiro Júnior Jl. Análises estatísticas no SAEG. Viçosa: Editora UFV; 2001. 301 p.

25. Erdman Jr WJ, Allen L H, Atkinson SA, Barr SI, Caballero B, Miller S A, et al. Panel on dietary reference intakes for electrolytes and water. In: DIETARY reference intakes for water, potassium, sodium, choride, and sulfate. Washington: Institute of Medicine, National Academy Press, 2003. p. 73-185. 